\title{
Self-similar aspects of fracture of metals in gigacycle fatigue loading
}

\author{
M.V. Bannikov ${ }^{\dagger}$ V.A. Oborin, O.B. Naimark \\ †mbannikov@icmm.ru
}

Institute of continuous media mechanics UB RAS, Perm, Russia

\begin{abstract}
Mechanisms of destruction of aviation motors advanced materials (aluminum alloy AMg6, pure titanium Grade-4 and titanium alloy TiAl6V4) are studied in terms of high- and gigacycle fatigue, including the influence of preliminary dynamic loading on the fatigue life. The theoretical understanding of the kinetics of fatigue crack growth in gigacycle loading conditions on the basis of incomplete self-similarity was shown. Samples of titanium grade Grade-4 were studied in three states: Original (average grain size of $25 \mathrm{um}$ ) and two submicrocrystalline (average grain size 150-200 nm) (UMP-1) and (UMP-2), obtained by equal-channel angular pressing in various conditions. Fatigue tests were carried out on Shimadzu USF-2000 ultrasonic fatigue testing machine in gigacycle fatigue regime and servohydraulic test machine Biss bi-00-100 in high cycle fatigue regime. Prestraining of AMg6 alloy was carried out on a split Hopkinson (Kolsky) bar. The increase of the fatigue strength of titanium alloys with decreasing grain size and significant decreasing in fatigue strength in gigacycle fatigue regime for preliminary deformed specimens for aluminum alloy were observed. Stages of initiation and propagation of fatigue cracks in the high-and gigacycle fatigue regimes were analyzed by quantitative profilometry of fracture surface. The description of the transition from particulate to the macroscopic destruction, including the initiation and growth of fatigue cracks is the basis for assessing the resource of materials in terms of fatigue. The scale invariance of the fracture surface morphology which allowed explaining the self-similar nature of crack growth under high- and gigacycle fatigue was established.
\end{abstract}

Keywords: Fracture, scaling, gigacycle fatigue, surface morphology, fractal analysis, Paris law.

\section{Автомодельные закономерности разрушения металлов при гигацикловом нагружении}

\author{
Банников М.В. ${ }^{\dagger}$ Оборин В.А., Наймарк О.Б. \\ †mbannikov@icmm.ru
}

Институт механики сплошных сред УрО РАН, ул. Ак. Королева 1, 614013, Пермь, Россия

\begin{abstract}
Закономерности разрушения перспективных материалов авиационного моторостроения (сплав алюминия АМг6, технический титан марки Grade-4 и титановый сплав ВT-6) исследуются в условиях много- и гигацикловой усталости, в том числе, с учетом влияния предварительного динамического нагружения на усталостную долговечность. Приведены теоретические представления о кинетике роста усталостной трещины в гигацикловом режиме нагружения на основе неполной автомодельности. Образцы из титана марки Grade-4 исследовались в трех состояниях: в исходном (средний размер зерна 25мкм) и двух субмикрокристаллических (средний размер зерна 150-200 нм) (УМ3-1) и (УМЗ-2), полученных методом равноканального углового прессования в различных условиях. Образцы подвергались усталостным испытаниям на резонансной усталостной машине Shimadzu USF-2000 в режиме гигацикловой усталости и сервогидравлической испытательной машине Biss bi-00-100 в режиме многоцикловой усталости. Предварительное деформирование сплава АМг6 осуществлялось на разрезном стержне Гопкинсона - Кольского. Установлено увеличение усталостной прочности титановых сплавов с уменьшением размеров зерен и значительное уменьшение усталостной прочности при гигацикловой усталости для предварительно деформированных образцов сплава алюминия. Стадии зарождения и распространения усталостной трещины в режимах много- и гигацикловой усталости анализировались по данным количественной профилометрии поверхностей разрушения. Описание характерных стадий перехода от дисперсного к макроскопическому разрушению, включая зарождение и развитие трещин, является основой для оценки временного ресурса изделий в условиях усталости. Установлена масштабная инвариантность рельефа поверхности разрушения, которая позволила предложить объяснение автомодельного характера роста трещины в условиях много- и гигацикловой усталости.
\end{abstract}

Ключевые слова: Разрушение, скейлинг, гигацикловая усталость, морфология поверхности, фрактальный анализ, закон Пэриса. 


\section{1. Введение}

Проблема оценки ресурса ответственных машиностроительных конструкций, в частности, авиационного моторостроения поставила ряд качественно новых задач фундаментального характера по оценке надежности современных материалов для значений, превышающих $10^{9}-10^{10}$ циклов нагружения, в области, так называемой, гигацикловой усталости [1]. Это связано с тем, что усталостный ресурс многих ответственных конструкций, работающих в режиме циклических нагрузок, превышает многоцикловый диапазон. В свою очередь гигацикловая область усталости, соответствующая числу циклов до разрушения $N \approx 10^{9}$, имеет ряд особенностей, для которых характерны качественные изменения как условий зарождения трещин, так и их распространения. При прохождении этого рубежа происходит смена механизмов зарождения и развития усталостных трещин.

Влияние случайных статических или динамических нагрузок на долговечность материалов в условиях гигацикловой усталости вызывает в настоящее время большой интерес в авиационном моторостроении в связи с необходимостью оценки усталостного ресурса, например, лопаток газотурбинных двигателей при соударении с твердыми частицами, получившую в западной литературе определение «foreign object damage» [2]. Решение данной проблемы предполагает использование результатов фундаментального характера, устанавливающих связь многомасштабной эволюции дефектных структур в материалах при различных условиях нагружения, в сочетании с современными методами структурного анализа, которые позволят прогнозировать кинетику зарождения и распространения трещин в материале, содержащем дефектны различных масштабных уровней, проводить оптимизацию структуры и подбор материалов, обладающих низкой чувствительностью долговечности к динамическим нагрузкам случайного характера в условиях много- и гигациклового нагружения.

\section{2. Кинетическое уравнение роста усталостной трещины}

Универсальность кинетических закономерностей, устанавливающих связь между скоростью роста усталостной трещины $d a / d N$ и изменением коэффициента интенсивности напряжений $\Delta K$, является предметом интенсивных экспериментальных и теоретических исследований. Степенные зависимости, впервые установленные в работах П. Пэриса [3] и известные как закон Пэриса, отражают автомодельный характер развития усталостных трещин, обусловленный нелинейным характером развития поврежденности в окрестности вершины трещины (process zone - «зона процесса»):

$$
\frac{\mathrm{da}}{\mathrm{dN}}=\mathrm{A}(\Delta \mathrm{K})^{\mathrm{m}} \text {, }
$$

где $A$ и $m$ экспериментально определяемые константы. Для широкого класса материалов и различных скоростей роста трещин в условиях многоцикловой усталости показатель степени $m$ близок к значениям 2-4.
Проявление автомодельных закономерностей роста трещины исследовалось методами теории подобия и размерностей [4-6]. Зависимость скорости роста трещины $d a / d N$ ( $a$ - длина трещины, $N$ - число циклов) определяется следующими параметрами:

$$
\frac{\mathrm{da}}{\mathrm{dN}}=\mathrm{F}\left(\Delta \mathrm{K}, \mathrm{E}, \mathrm{l}_{\mathrm{sc}}, \mathrm{L}_{\mathrm{pz}}\right)
$$

где $\Delta K-$ размах коэффициента интенсивности напряжений; $E-$ модуль Юнга; $l_{s c}$ - минимальный пространственный масштаб в окрестности вершины трещины (зоны процесса разрушения), на котором начинают проявляться масштабно-инвариантные закономерности рельефа поверхности разрушения, $L_{p z}-$ масштаб зоны процесса разрушения в вершине трещины. Значения $L_{p z}$ и $l_{s c}$ определялись экспериментально с помощью масштабного инварианта (показателя Херста), полученного из анализа функции корреляции профилей поверхности разрушения. Следуя П-теореме $[4,6]$ в безразмерном виде функцию (2) в рамках независимых переменных можно представить в виде:

$$
\frac{\mathrm{da}}{\mathrm{dN}}=\Phi\left(\frac{\Delta \mathrm{K}}{\mathrm{E} \sqrt{1_{\mathrm{sc}}}}, \frac{\mathrm{L}_{\mathrm{pz}}}{\mathrm{l}_{\mathrm{sc}}}\right) .
$$

Оценка значений $\Delta \mathrm{K} / \mathrm{E} \sqrt{1_{\mathrm{sc}}}<<1$ и $\mathrm{L}_{\mathrm{pz}} / 1_{\mathrm{sc}}>>1$ позволяет предположить промежуточно-асимптотический характер кинетики роста трещины и записать (3) в виде:

$$
\frac{\mathrm{da}}{\mathrm{dN}}=1_{\mathrm{sc}}\left(\frac{\Delta \mathrm{K}}{\mathrm{E} \sqrt{1_{\mathrm{sc}}}}\right)^{\alpha}\left(\frac{\mathrm{L}_{\mathrm{pz}}}{\mathrm{l}_{\mathrm{sc}}}\right)^{\beta},
$$

где $\alpha$ и $\beta$ - степенные показатели, отражающие промежуточно-асимптотический характер кинетики роста трещины от безразмерных переменных. Вводится параметр $\Delta K_{\text {eff }}=\Delta K\left(L_{p z} / l_{s c}\right)^{\beta / \alpha}$, который позволит записать уравнение (4) в виде:

$$
\frac{\mathrm{da}}{\mathrm{dN}}=1_{\mathrm{sc}}\left(\frac{\Delta \mathrm{K}_{\mathrm{eff}}}{\mathrm{E} \sqrt{1_{\mathrm{sc}}}}\right)^{\alpha} .
$$

Экспериментальное представление уравнения (5) для стали R4 отражено в работе [7].

\section{3. Усталостные испытания титана и его сплавов в режиме много- и гигацикловой усталости}

Исследовались образцы из сплава титана ВТ-6 (Ti6Al4V) и технически чистого титана марки Grade 4 в различных состояниях микроструктуры (Рис.1). В исходном крупнокристаллическом состоянии средний размер зерна составлял 25 мкм; в ультрамелкозернистом (субмикрокристаллическом) состоянии УМЗ-1 средний размер зерен - 150-200 нм; в ультрамелкозернистом состоянии УМЗ-2 средний размер зерна составлял 200 нм. Измельченное зерно было получено с помощью равноканального углового прессования в следующих режимах: УМЗ-1 $\mathrm{T}=450{ }^{\circ} \mathrm{C}, 8$ проходов, волочение с 14 до 9 мм при $\mathrm{T}=200{ }^{\circ} \mathrm{C}$; УМЗ-2 $\mathrm{T}=450{ }^{\circ} \mathrm{C}, 4$ прохода, теплая прокатка с 12 до 8 мм при $\mathrm{T}=350^{\circ} \mathrm{C}$.

По данным просвечивающей электронной микроскопии обнаружено, что в состоянии УМЗ-1 микроструктура более однородная, зерна имеют равноосную форму 


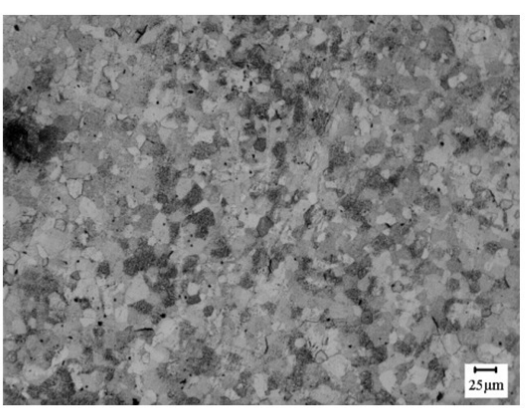

a

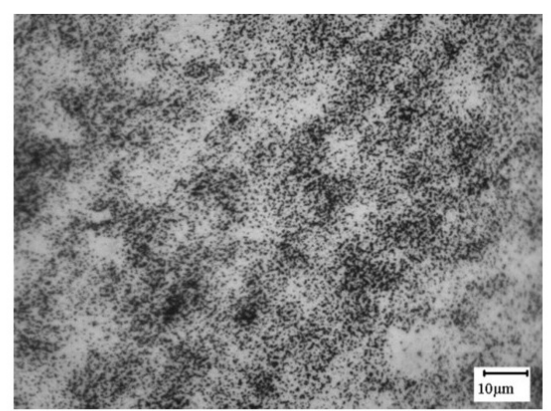

b

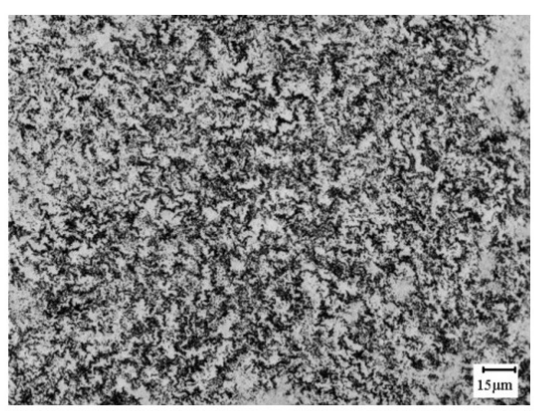

c

Pис. 1. Структура Ti Grade-4, изображения микрошлифов поверхности, полученные оптическим металлографическим микроскопом: (a) исходное состояние (размер зерна 25 мкм); и электронным микроскопом (b) Состояние УМЗ-1 (размер зерна 150-200 нм); (с) Состояние УМЗ-2 (размер зерна 200 нм).

Fig. 1. Structure of Ti Grade-4: a) optical microscopy image of initial state (grain size $25 \mu \mathrm{m}$ ); and electronic microscope b) image of SMC-1 state (grain size $\sim 150-200 \mu \mathrm{m}$ ); c) image of SMC-2 (grain size $\sim 200 \mu \mathrm{m}$ ).

как в поперечном, так и в продольном сечении. В УМЗ2 в продольном сечении прутка наблюдается развитая металлографическая текстура, которая характеризуется удлиненными зернами с дислокационной субструктурой в результате прокатки после РКУП.

Из прутков исследуемых материалов вырезались образцы в виде песочных часов с определенными размерами для каждого материала (рис.2). Данные размеры, зависящие от упругих модулей и плотности материала, определялись по аналитическим формулам [7], обеспечивающие условия стоячей волны и резонанса испытательной системы.

Испытания на усталость проводились на испытательной машине резонансного типа (Shimadzu USF2000) с коэффициентом асимметрии цикла $\mathrm{R}=-1$. Испытательная машина состоит из следующих основных частей: генератора, преобразующего частоту 50 Гц в ультразвуковой электрический синусоидальный сигнал с частотой 20 кГц; пьезоэлектрического преобразователя, генерирующего продольные ультразвуковые волны в механическое воздействие частотой 20 кГц; ультразвукового волновода, формирующего максимальную амплитуду механического напряжения в рабочей (средней) части образца. Изменение частоты на 0,5 кГц соответствовало появлению трещины с характерным размером 2 мм.

Результаты испытаний изображены на сводном графике (Рис. 3) в сопоставлении с литературными данными из работы [8] для сплава Ti6Al4V. Усталостное

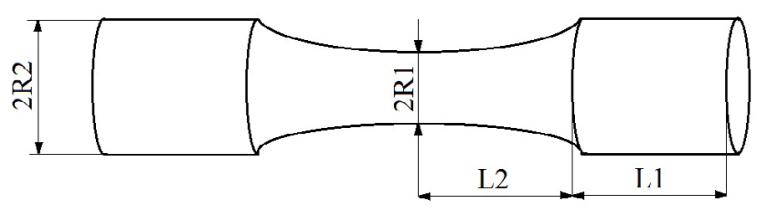

Рис. 2. Геометрия образцов. Размеры L1,L2,R1,R2 подбираются для выполнения условия резонанса по формулам, описанным в [8].

Fig. 2. The geometry of samples. Dimensions L1, L2, R1, R2 are selected to satisfy the condition of resonance by the formulas described in [8]. разрушение сплава ВТ-6 на базе испытаний $10^{9}$ циклов произошло при амплитуде напряжения 495 МПа. Разрушения Ti Grade-4 на базе $10^{9}$ циклов нагружения произошли при амплитудах напряжений $275 \mathrm{MПа} \mathrm{для} \mathrm{исходно-}$ го состояния и 375 МПа и 340 МПа для состояний УМЗ-1 и УМЗ-2 соответственно. Результаты усталостных испытаний сплава титана BT-6 (Ti6Al4V) в гигацикловом режиме нагружения хорошо согласуются с данными работ [8-9]. Прослеживается влияние микроструктуры материала на его усталостные характеристики в режиме гигацикловой усталости. В поликристаллическом состоянии структуры с размером зерна 25 мкм титан уступает по усталостной прочности субмикрокристаллической модификации. Также прослеживается небольшая разница в усталостных свойствах двух субмикрокристаллических состояниях: Ti Grade-4 УМ3-1 с равновесными границами зерен проявляет более высокие усталостные свойства по сравнению с состоянием УМЗ-2, где состояние границ зерен менее однородно.

Для установления механизмов разрушения в режиме гигацикловой усталости поверхности разрушения всех образцов исследовались с применением оптической микроскопии и профилометрии.

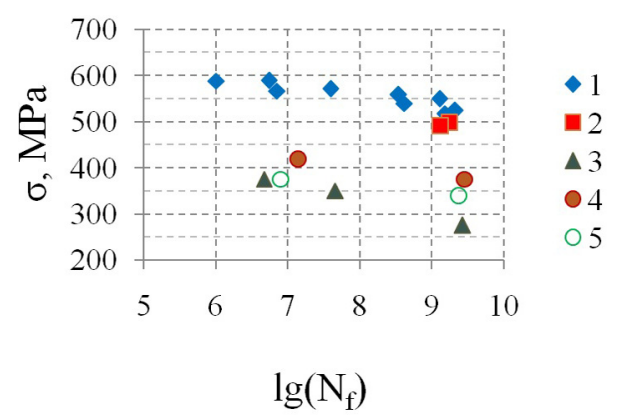

Рис. 3. Результаты испытаний на гигацикловую усталость: $1-$ данные по усталостной долговечности для сплава Ti6Al4V [8]; 2 - Сплав ВT-6; 3 - Ti Grade-4 в исходном состоянии; $4-\mathrm{Ti}$ Grade-4 в ультрамелкозернистом состоянии УМ3-1; 5 - Ті Grade-4 в ультрамелкозернистом состоянии УМЗ-2.

Fig. 3. Fatigue curve data for investigated materials: $\sigma-$ applied mean stress, $\mathrm{N}_{\mathrm{f}}$-number of cycles to failure. $1-$ Ti6Al4V Bathias [8]; 2 - Ti6Al4V; 3 - Ti Grade-4 initial state; 4 - Ti Grade-4 SMC1 state; 5 - Ti Grade- 4 SMC-2 state. 
В образцах из титана и его сплавов, разрушенных изнутри с характерным видом излома "fish-eye", обнаружены характерные области вокруг очага инициирования трещины диаметром 100 мкм для чистого титана марки grade-4 и 300 мкм для сплава ВТ-6 (рис. 4), которые обладают высокой шероховатостью. Остальная область, которая охватывает большую часть поверхности разрушения, является более гладкой, чем первая.

Для исследования масштабно-инвариантных закономерностей из двумерного профиля поверхности разрушения, полученного на интерферометре New View, вырезались одномерные профили различной длины, охватывающие как обе характерные области шероховатости, так и ограниченные только одной зоной (синие и красные линии на рис.4). Профили анализировались функцией корреляции $K(r)$ :

$$
\mathrm{K}(\mathrm{r})=\left\langle(\mathrm{z}(\mathrm{x}+\mathrm{r})-\mathrm{z}(\mathrm{x}))^{2}\right\rangle_{\mathrm{x}}^{1 / 2} \propto \mathrm{r}^{\mathrm{H}},
$$

где $K(r)$ представляет собой усредненную разность значений высот рельефа поверхности $z(x+r)$ и $z(x)$ на окне размером $r, H$ - показатель Херста (показатель шероховатости) [7,10-13].

Выбирались профили, направление которых соответствовали распространению усталостной трещины (Рис. 3). Функция корреляции, построенная по профилям, включающих обе характерные зоны, имеет два линейных участка с изломом на масштабе, соответствующем изменению механизмов формирования рельефа поверхности разрушения, тогда как функции, построенные по профилям внутри зон, имели только один линейный участок. При этом значения масштабного инварианта $H$, полученные на профилях с одним линейным участком, соответствовали значениям, полученным на профилях с двойным наклоном.

В результате анализа масштабно-инвариантных закономерностей было установлено, что зона вокруг очага разрушения связана с накоплением микроповреждений и формированием усталостной трещины. По мере ци-

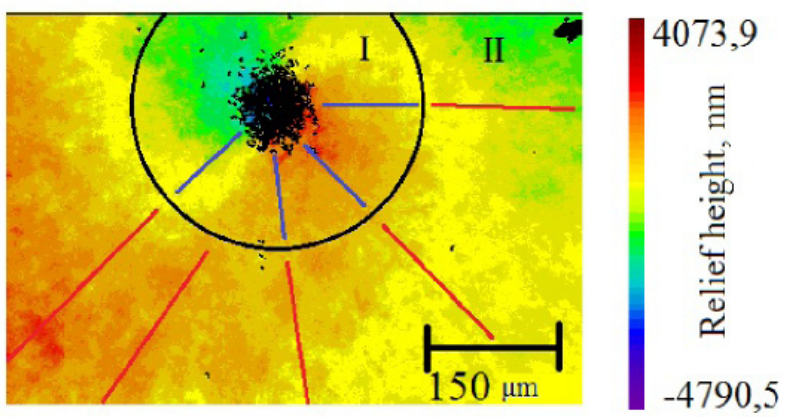

Рис. 4. Изображение очага усталостной трещины и характерной зоны повышенной шероховатости вокруг него, полученное на интерферометре New View 5010. Красными и синими линиями показаны исследуемые одномерные профили внутри зоны I и зоны II.

Fig. 4. Image of the fatigue crack origin in cylindrical samples (Ti6Al4V) by interferometer New View 2-D. Marked zones I and II are the areas of crack initiation and propagation, respectively. Solid lines are cross-sections of surface roughness used for the estimation of scale invariance (the Hurst exponent). клического нагружения дефекты накапливаются и образуют усталостную трещину критического размера, начиная с которого ее рост подчиняется закону Пэриса, о чем свидетельствует изменение шероховатости поверхности и как следствие изменение значения показателя Херста.

\section{4. Оценка надежности алюминиевого сплава АМг6 при предварительном динамическом нагружении}

Предварительное нагружение образцов из сплава алюминия и магния АМг6 осуществлялось динамическим растяжением на разрезном стержне Гопкинсона-Кольского при скоростях деформации до $\sim 10^{3} \mathrm{c}^{-1}$, после чего образцы подвергались циклической нагрузке при комнатной температуре, с последующим изучением фрактографии изломов с помощью интерферометра-профилометра New View 5010.

Уровень приложенных напряжений позволял проводить исследования усталостного ресурса до значений, соответствующих $10^{10}$ циклов. Исследование долговечности проводилось при уровнях напряжений (105 - 160 МПа), соответствующих критическому количеству циклов $\sim 10^{10}$, оцениваемых для материалов в исходном (недеформированном) состояниях для условий гигацикловой усталости. Результаты усталостных испытаний приведены в таблице 1 .

Из таблицы видно, что при средней амплитуде нагружения 154 МПа происходит смена механизмов и образец АМг6 разрушается по достижению $10^{9}$ циклов нагружения (гигацикловая усталость), тогда как уже при 155 МПа он разрушается значительно раньше $\left(4,2 \cdot 10^{6}\right)$. После предварительного динамического нагружения разрушение по механизму многоцикловой усталости происходит при значительно меньших амплитудах среднего напряжения (105-130 МПа).

Таблица 1. Результаты усталостных испытаний сплава АМг6 с предварительным нагружением

Table 1. Results of fatigue tests of AMg6 alloy with preliminary loading

\begin{tabular}{|c|c|c|c|}
\hline $\begin{array}{c}\text { Удлинение } \\
(\text { мм })\end{array}$ & $\begin{array}{c}\text { Скорость } \\
\text { удника } \\
(\mathrm{m} / \mathrm{c})\end{array}$ & $\begin{array}{c}\sigma, \\
\text { Среднее } \\
\text { напряжение } \\
(\mathrm{MПа})\end{array}$ & $\begin{array}{c}\Delta \mathrm{N}, \\
\text { циклы до } \\
\text { разрушения }\end{array}$ \\
\hline $\begin{array}{c}\text { Elongation } \\
(\mathrm{mm})\end{array}$ & $\begin{array}{c}\text { Projectile } \\
\text { velocity } \\
(\mathrm{m} / \mathrm{s})\end{array}$ & $\begin{array}{c}\text { (Mean } \\
\text { stress }(\mathrm{MPa})\end{array}$ & $\begin{array}{c}\Delta \mathrm{N}, \text { cycles } \\
\text { to failure }\end{array}$ \\
\hline 0 & 0 & 160 & $6,93 \cdot 10^{+6}$ \\
\hline 0 & 0 & 155 & $4,2 \cdot 10^{+6}$ \\
\hline 0 & 0 & 154 & $1,41 \cdot 10^{+9}$ \\
\hline 0,89 & 28,4 & 130 & $7,33 \cdot 10^{+6}$ \\
\hline 1,77 & 40,3 & 120 & $7,82 \cdot 10^{+8}$ \\
\hline 1,27 & 32,1 & 120 & $5,72 \cdot 10^{+7}$ \\
\hline 2,21 & 40,3 & 105 & $5,83 \cdot 10^{+6}$ \\
\hline
\end{tabular}


Поверхностный рельеф разрушенных образцов регистрировался с помощью интерферометра-профилометра высокого разрешения New-View (при увеличении х2000) и затем анализировался методами фрактального анализа для определения условий коррелированного поведения многомасштабных дефектных структур, с которым связывалось распространение трещины.

При усталостных испытаниях наблюдались два типа разрушения образцов. Первый, когда образцы разрушались непосредственно во время эксперимента. Второй, когда образцы, обладающие явными признаками разрушения (сильное изменение резонансной частоты испытаний, выход усталостной трещины на поверхность), уже были не способны продолжать выдерживать усталостную нагрузку в резонансной частоте. Поверхность разрушения образцов первого и второго типа «вскрывалась» охлаждением образцов жидким азотом и последующим доломом. Предполагается, что поверхность разрушения в режиме гигацикловой усталости уже сформировалась в процессе эксперимента и занимает большую часть поверхности разрушения, что сопровождается изменением резонансной частоты испытаний.

При разрушении цилиндрических образцов за число циклов, соответствующих многоцикловой усталости $\left(10^{6}-10^{7}\right)$, трещина образуется с поверхности образца (Рис. 5a). При разрушении на базе $10^{8}$ циклов и более трещина образуется внутри образца, и на поверхности разрушения видна характерная для такого режима усталости область излома - «рыбий глаз» («fish-еуе»), в центре которой находится очаг разрушения, окруженный областью с фрагментированной (субмикрокристаллической) структурой (светлая область), рис. $5 \mathrm{~b}$.

Области сканирования распределялись в зоне роста усталостной трещины (рис. 5a) и анализировались одномерные образы-срезы рельефа поверхности в радиальном направлении по отношению к границе раздела между зонами 1 и 3. В пределах каждого «окна» анализировались 12 одномерных «срезов», обеспечивая представительность данных о структуре рельефа, индуцированного дефектами, с вертикальным разрешением $\sim 0,1$ нм и горизонтальным $\sim 0,1$ мкм.

Для определения минимального (критического) масштаба $l_{s c}$ соответствующему установлению длинно-корреляционных взаимодействий в ансамблях дефектов, использовался метод определения показателя Херста
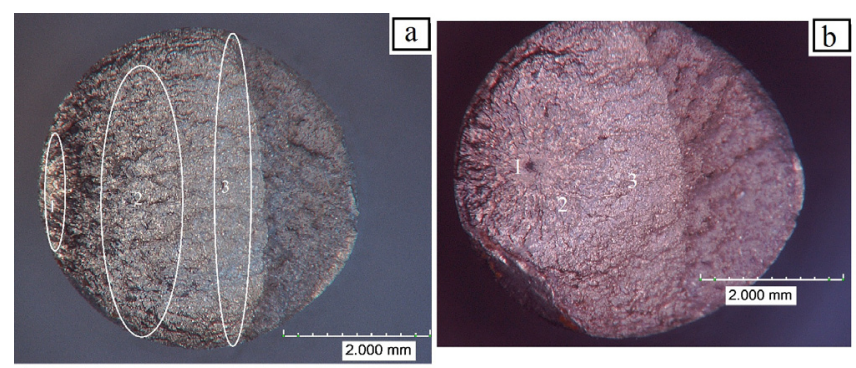

Рис. 5. Характерный рельеф поверхности зоны усталостного разрушения: (a) при многоцикловой усталости, (b) при гигацикловой усталости.

Fig. 5. Characteristic surface relief of a fatigue fracture zone: high cycle fatigue (a), gigacycle fatigue (b).
[10-11]. По одномерным профилям рельефа поверхности разрушения вычислялась функция $K(r)$ по формуле (6).

Представление функции $K(r)$ в логарифмических координатах (рис. 6а) в соответствие с соотношением (6) позволяет провести оценку критического масштаба $l_{s c}$ (рис. 6b). Значение нижней границы масштаба скейлинга принималось за значение критического масштаба $l_{s c}$ значение верхней границы принималось за значение масштаба связанного с зоной процесса $L_{p z}$ - областью коррелированного поведения дефектных структур (рис. 5b).

Сравнение скейлинговых характеристик образцов, нагруженных в условиях много- и гигацикловой усталости, позволил установить существенное уменьшение диапазона пространственных масштабов (0,5-10,9 мкм), на которых показатель Хёрста остается постоянным для динамически нагруженных образцов в зоне «fish-eye», что находится в соответствие с результатами [12-13].

\section{5. Заключение}

Анализ данных морфологии поверхности позволил установить масштабную инвариантность рельефа поверхности разрушения и связать ее с автомодельным характером распространения усталостной трещины в режиме гигацикловой усталости.

Сравнительный анализ масштабных инвариантов в зоне «fish-еуе», нагруженных в условиях много- и гигацикловой усталости, позволил установить существенное уменьшение диапазона пространственных масштабов, на которых показатель Хёрста остается постоянным для динамически нагруженных образцов. Этот результат подтверждает наше предположение о значимой роли характеристических масштабов $L_{p z}$ и $l_{s c}$ определяющих автомодельный характер роста усталостной трещины.
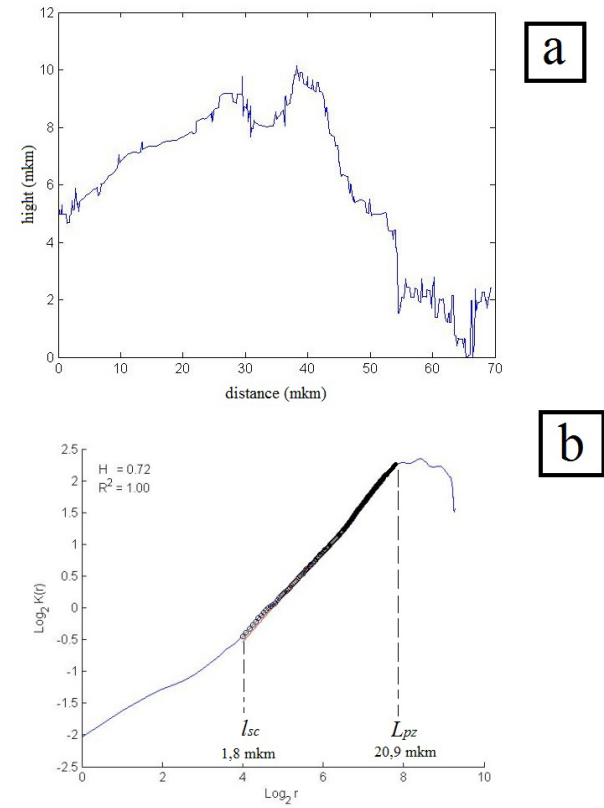

Рис. 6. Характерный: (а) одномерный профиль в зоне 1; (b) вид зависимости $\ln K(r)$ от $\ln (r)$ для зоны 1.

Fig. 6. Characteristic one-dimensional profiles for zone 1 (a), plot $\ln K(r)$ vs. $\ln (r)$ for zone 1 (b). 
Благодарность. Работа выполнена при поддержке гранта РНФ 14-19-01173.

Acknowledgements. This study was supported by the Russian Science Foundation, project No. 14-19-01173.

\section{Литература / References}

1. L.R. Botvina. Fracture: Kinetics, Mechanisms, General Laws. Moscow, Nauka. (2008) 334 p. (in Russian) [Л. Р. Ботвина. Разрушение: кинетика, механизмы, общие закономерности. Москва, Наука. 2008. 334 с.]

2. J. O. Peters. Eng. Fract. Mech. 67, 193-207 (2000).

3. P. Paris, D. Lados, H. Tad. Engineering Fracture Mechanics. 75, 299-3052 (2008).

4. G.I. Barenblatt . Int.J. of Fracture. 138, 19-35 (2006).

5. R.O. Ritchie. Int. J. of Fracture. 132, 197-203 (2005).
6. M. Ciavarella, M. Paggi, A. Carpinteri J.Mech.Phys.Solids 56(2008)3416-3432.

7. Oborin V.A. Bannikov M.V. Naimark O.B.Palin-Luc T., Technical Physics Letters, 36 (11), 1061-1063 (2010).

8. C. Bathias, P. C. Paris. Gigacycle Fatigue in Mechanical Practice . Marcel, Dekker Publisher Co. (2005) 328 p.

9. H. Mughrabi. Int. J. Fatigue. 28, 1501-1508 (2006).

10. E. Bouchaud. J. Phys. Condens. Matter. 9, 4319- 4344 (1997).

11. C. Froustey, O. Naimark, M. Bannikov, V.Oborin. European Journal of Mechanics A/Solids. 29, 1008-1014 (2010).

12. M. Bannikov, V. Oborin, O. Naimark, and C. Froustey, Technical Physics Letters.37( 3), 241-243 (2011).

13. Froustey C., Naimark O., Bannikov M., Oborin V. / European Journal of Mechanics A/Solids. 29, 1008-1014, (2010). 